\title{
Wie lockt man Zeitschriftenautoren?
}

\author{
Joachim Hertzberg
}

Eingegangen: 1. April 2010 / Angenommen: 8. April 2010 / Online publiziert: 17. April 2010

(C) Springer-Verlag 2010

\section{Liebe Leserin, lieber Leser!}

In Editorials der Communications of the ACM ging es jüngst - zuletzt im März 2010 - wieder um das alte Thema, ob es eigentlich sinnvoll sei, dass Informatiker ihre wichtigsten Ergebnisse oft auf Tagungen publizieren, und nicht in Zeitschriften wie der Rest der Wissenschaftswelt. Keine Angst, ich will die Diskussion hier nicht wiederbeleben: Zwar lese ich zuweilen Editorials (Sie offenbar auch, willkommen!), aber ich glaube nicht daran, gewachsene Fächerkulturen durch noch so gut geschriebene Vorworte zu verändern. Auch ich finde es in vieler Hinsicht dumm, dass wir Informatiker uns eine andere Publikationskultur leisten als alle anderen. Aber ich habe mich daran gewöhnt, diese Marotte Fachfremden zu erklären, ich mache sie mit und freue mich in fröhlicher Inkonsistenz an lebendigen Tagungen und edlen Tagungsbänden.

Das März-Editorial der Communications lief darauf hinaus: Damit die Informatik die Publikationskultur anderer Fächer übernimmt, müsse erst die Kultur und Qualität ihrer Zeitschriften den Standard der anderen erreichen, also zum Beispiel die gleiche Geschwindigkeit erzielen bei der Bearbeitung und Veröffentlichung von Einreichungen. Ich bin nicht sicher, dass Informatik-Zeitschriften schlechter als andere funktionieren. Doch wie auch immer: Publikation in einer Zeitschrift ist natürlich umso attraktiver, je kompetenter sie begutachtet, je weiter sie ausstrahlt und je schneller angenommene Manuskripte erscheinen - idealerweise übrigens schneller als angenommene Tagungsbeiträge.

J. Hertzberg $(\bowtie)$

Institut für Informatik, Universität Osnabrück, Albrechtstraße 28, 49076 Osnabrück, Deutschland e-mail: joachim.hertzberg@uos.de url: http://www.inf.uos.de/hertzberg/
Damit sind wir bei den neuen Regeln für die KIZeitschrift, die seit diesem Jahrgang gelten. Ab jetzt erscheinen alle Beiträge umgehend nach Eingang der Endfassung (bzw. nach Fertigstellung des Heft-Schwerpunkts, wenn sie zu einem solchen gehören) online bei Springer Link - oft also lang vor Erscheinen des gedruckten Hefts. Beiträge werden ab sofort bei DBLP, Google Scholar, OCLC und Summon indiziert. Die Prozedur zur Erhebung des Citation Index der KI-Zeitschrift ist angestoßen. Falls Sie für die KIZeitschrift gutachten, werden Sie feststellen, dass Sie nach Annahme eines Gutachten-Auftrags eine 21-Tage Frist gesetzt bekommen; an diese Frist werden Sie gegebenenfalls erst freundlich automatisch erinnert, dann automatisch gemahnt - und notfalls würde sich dann einer der Herausgeber um Sie kümmern ...

Kurzum: Die KI-Zeitschrift ist dabei, ihre Reichweite und ihre Geschwindigkeit signifikant zu erhöhen. Dabei helfen natürlich die Infrastruktur und die Erfahrung des Springer Verlags. Auch ohne die Herausgeber würde es nicht klappen; daher freue ich mich, seit diesem Jahr dabei zu sein und an der „Neuen KI-Zeitschrift" mitzuarbeiten.

Am Ende liegt die Qualität einer Zeitschrift - Verlag hin, Herausgeber her - bekanntlich an der Qualität der Manuskripte, die eingereicht werden. Publizieren Sie weiter auf internationalen Tagungen, ich werde das auch tun. Doch denken Sie zuweilen an die KI-Zeitschrift! Und haben Sie hier ein interessantes Papier gelesen, trauen Sie sich, es zu zitieren! Die lokale Infrastruktur für die KI ersetzt nicht die internationale; aber es schadet auch nicht, sie zu nutzen und zu pflegen.

Freundliche Grüße

Joachim Hertzberg 


\section{Schwerpunkt - Cognition for Technical Systems}

The research area Cognition for Technical Systems investigates the realization of cognitive capabilities such as perception, learning, reasoning, planning, and execution for technical systems including humanoid robots, flexible manufacturing systems, and mixed teams of humans and robots. One of the ultimate goals is, as Brachman puts it in the context of general cognitive systems, to turn technical systems into "ones that can reason using substantial amounts of appropriately represented knowledge, learn from their experience so that they perform better tomorrow than they did today, explain themselves and be told what to do, be aware of their own capabilities and reflect on their own behaviour, and respond robustly to surprise."

A technical system that is cognitive in this sense will be more reliable, flexible, and adaptive. These kinds of systems ease interaction and cooperation with humans.

This special issue of KI focuses on the role of Artificial Intelligence in cognitive technical systems, on methods of Artificial Intelligence that apply well to cognitive technical systems, and on the challenges that cognitive technical systems present to Artificial Intelligence research.

We solicit reports on current research projects, summaries of doctoral theses, book reviews and conference reports. Interested authors should contact the guest editor as soon as possible:

\section{Prof. Dr. Michael Beetz}

Fakultät für Informatik

Technische Universität München

Boltzmannstr. 3

85748 Garching b. München

Deutschland

beetz@in.tum.de

\section{Schwerpunkt - Simultaneous Localization and Mapping}

Für einen Roboter - also einen intelligenten Agenten mit physischer Präsenz - ist die Wahrnehmung seiner Umgebung eine erste Grundvoraussetzung. In einem zweiten Schritt stellt sich die Aufgabe, diese lokalen Wahrnehmungen zu einer globalen Repräsentation seines Arbeitsraumes, also einer Karte, zusammenzusetzen. Ende der 1980er Jahre hat sich die Erkenntnis durchgesetzt, dass dies ein Huhnund-Ei-Problem ist, weil das Bilden einer Karte zu wissen erfordert, wo der Roboter ist und den Roboter zu lokalisieren eine Karte erfordert. Mit dieser Erkenntnis war das Simultaneous Localization and Mapping (SLAM) Problem geboren.

Seitdem hat das Feld eine gewisse Reife erlangt. Anlass genug für das Schwerpunktheft SLAM zurückzublicken und zu fragen „Was wurde erreicht? Ist SLAM gelöst?“ und nach vorne zu blicken und neue Entwicklungen von SLAM im größeren Kontext der KI zu betrachten:

- Repräsentation der Unsicherheit, was beobachtet wurde (Datenassoziation)

- Verknüpfung sensoriell wahrgenommener metrischer und semantischer Information

- Aktionsplanung in einer unsicheren Karte, z. B. zur Exploration

- Topologische und metrische Karten in SLAM und der Bezug dazwischen

- Visuelles SLAM in 3D mit einer Kamera als Sensor

- Reale und industrielle Anwendungen von SLAM

Von Interesse sind Fachbeiträge, Berichte über laufende Forschungsprojekte, Dissertationen, Interviews, Schlagwörter, Buchbesprechungen und Tagungsberichte. Beiträge können in deutscher oder englischer Sprache verfasst werden. Weitere Informationen finden sich unter http://www.informatik. uni-bremen.de/agebv/de/KI-Schwerpunkt-SLAM.

Interessierte Autorinnen und Autoren werden gebeten, sich möglichst bald an den Gastherausgeber zu wenden.

\section{Prof. Dr. Udo Frese}

Juniorprofessor für Echtzeitbildverarbeitung

Universität Bremen

Enrique-Schmidt-Straße 5

28359 Bremen

Deutschland

ufrese@informatik.uni-bremen.de

\section{Schwerpunkt - 25 Jahre KI und KI-Zeitschrift}

Seit 1987 erscheint die Zeitschrift „Künstliche Intelligenz“ in einem Verlag, der Vorgänger „KI-Rundbrief“ wurde als Manuskript von Universitäten bzw. Forschungseinrichtungen kopiert und verteilt. In diesem ,Jubiläumsheft“ wird unter verschiedenen Aspekten auf diese 25 Jahre KI und KIZeitschrift zurückgeblickt.

Darüber hinaus ist geplant, auch die nähere und fernere Zukunft der KI und der Zeitschrift zu beleuchten.

Interessierte Autorinnen und Autoren werden gebeten, sich möglichst bald an den Gastherausgeber zu wenden. Es sind zur Vergangenheit und Zukunft der KI und ihrer Zeitschrift Beiträge aller Art erwünscht!

\section{Dr. Andreas Günter}

HITeC e.V./Uni Hamburg

Vogt-Kölln-Str. 30

22527 Hamburg

Deutschland

Tel: 040/42883-2456

guenter@informatik.uni-hamburg.de 


\section{Schwerpunkt - Off-road Robotik}

Als Off-road Roboter werden Systeme bezeichnet, die autonom bzw. teilautonom in rauem, natürlichem und unebenen Gelände agieren. Im Gegensatz zur Forschung im Bereich der kognitiven Automobile zeichnet sich die Off-road Robotik durch die Unstrukturiertheit der Umwelt, die Variabilität der Umgebungsbedingungen, die zu berücksichtigende Dreidimensionalität und die hohe Hindernisdichte aus. Die Unstrukturiertheit und die Variabilität der Umwelt erschweren zum einen die Erkennung der Umweltgegebenheiten und zum anderen die Navigation solcher Systeme.

Im Rahmen des Schwerpunkts Off-road Robotik werden Beiträge zu folgende Bereichen vorgestellt:
- Umweltrepräsentation und Karten

- Situationserkennung und die Geländeeinschätzung

- Lokale und globale Navigationsverfahren

- Anwendungsprojekte

Interessierte Autorinnen und Autoren werden gebeten, sich an den Gastherausgeber zu wenden.

\section{Prof. Dr. Karsten Berns}

AG Robotersysteme

Fachbereich Informatik

Technische Universität Kaiserslautern

Postfach 3049

67653 Kaiserslautern

Deutschland

berns@informatik.uni-kl.de 